\title{
Joint analysis of the energy spectrum of ultra-high-energy cosmic rays measured at the Pierre Auger Observatory and the Telescope Array
}

\author{
Yoshiki Tsunesada ${ }^{a, b *}$ on behalf of the Pierre Auger ${ }^{c}$ and the Telescope Array ${ }^{d}$ \\ Collaboration \\ (a complete list of authors can be found at the end of the proceedings) \\ ${ }^{a}$ Graduate School of Science, Osaka City University \\ ${ }^{b}$ Nambu Yoichiro Institute for Theoretical and Experimental Physics, Osaka City University \\ ${ }^{c}$ Observatorio Pierre Auger, Av. San Martín Norte 304, 5613 Malargüe, Argentina \\ ${ }^{d}$ Telescope Array Project, 201 James Fletcher Bldg, 115 S. 1400 East, Salt Lake City, UT 84112-0830, USA \\ E-mail: tsunesada@osaka-cu.ac.jp, spokespersons@auger.org, \\ ta-icrc@cosmic.utah.edu
}

\begin{abstract}
The measurement of the energy spectrum of ultra-high-energy cosmic rays (UHECRs) is of crucial importance to clarify their origin and acceleration mechanisms. The Pierre Auger Observatory in Argentina and the Telescope Array (TA) in the US have reported their measurements of UHECR energy spectra observed in the southern and northern hemisphere, respectively. The region of the sky accessible to both Observatories $([-15,+24]$ degrees in declination) can be used to crosscalibrate the two spectra. The Auger-TA energy spectrum working group was organized in 2012 and has been working to understand the uncertainties in energy scale in both experiments, their systematic differences, and differences in the shape of the spectra. In previous works, we reported that there was an overall agreement of the energy spectra measured by the two observatories below $10 \mathrm{EeV}$ while at higher energies, a remaining significant difference was observed in the common declination band. We revisit this issue to understand its origin by examining the systematic uncertainties, statistical effects, and other possibilities. We will also discuss the differences in the spectra in different declination bands and a new feature in the spectrum recently reported by the Auger Collaboration.
\end{abstract}

$37^{\text {th }}$ International Cosmic Ray Conference (ICRC 2021)

July 12 th - 23rd, 2021

Online - Berlin, Germany

\footnotetext{
*Presenter
} 


\section{Introduction}

The existence of protons and nuclei with joule-scale kinetic energies - up to $10^{20} \mathrm{eV}-$, known as ultra-high energy cosmic rays (UHECRs), is one of the most intriguing unsolved problems in modern astrophysics. Discovering the origin of these particles would allow us to understand the most energetic phenomena occurring in the universe. The precise measurement of their energy spectrum, corresponding to the differential intensity $d I / d E$ of the particles, is of particular importance because its absolute scale and its shape are closely related to the production rate in the sources, which in turn is related to the acceleration mechanisms at such extreme energies, as well as to the spatial distribution of the sources, which shapes the propagation that cosmic rays have to perform to be detected on Earth. The spectrum of cosmic rays above $10^{18} \mathrm{eV}$ is known to be well described by a series of power laws, $d I / d E \propto E^{-\gamma}$, with a spectral index $\gamma \sim 3.2-3.3$ below the "ankle" feature around $5 \times 10^{18} \mathrm{eV}$, hardening to $\gamma \sim 2.6-2.7$ beyond the ankle, and steepening to $\gamma \sim 5$ beyond $\simeq 5 \times 10^{19} \mathrm{eV}$. Recent observations at the Pierre Auger Observatory and at the Telescope Array have revealed an additional spectral feature, with the capture of a spectral index change around $10^{19} \mathrm{eV}$ from $\gamma \sim 2.6-2.7$ to $\gamma \sim 3$.

The arrival of UHECR on the Earth is so rare, about one event per square kilometer per year, that huge detection areas and long observation times are necessary. The two currently operational observatories, the Pierre Auger Observatory in Argentina and the Telescope Array (TA) in the United States, cover areas of $3000 \mathrm{~km}^{2}$ and $700 \mathrm{~km}^{2}$, respectively. Similar detection techniques are used by the two observatories, but their detailed characteristics and data reconstruction methods are quite different. A joint working group of the two experiments was formed in 2012 to discuss the technical details of the data analyses, and the results of its activities were reported in the UHECR and ICRC conference series [1,2]. In this contribution, we revisit the details of the Auger and TA data analysis, the systematic uncertainties in the energy determination, and the agreements and differences in the energy spectrum obtained by the two experiments.

\section{Auger and TA detectors}

Two types of extensive air shower detection techniques are used at the Auger Observatory and TA. Arrays of surface detectors (SDs), sampling the lateral profile of the showers at ground level, provide us with very large collection areas and exposure thanks to an almost $100 \%$ duty cycle. The SD arrays are overlooked by fluorescence detectors (FDs), sensitive to the fluorescence light isotropically emitted by atmospheric molecules along the shower particle track. The FD enables an almost calorimetric measurement of the cosmic-ray energy, and therefore an energy determination of the showers that is almost insensitive to details of hadronic interactions of cosmic rays in the atmosphere, which is rather uncertain because the center-of-mass energy of cosmic ray - atmospheric interactions is beyond the present accelerator energies.

The Auger Observatory is located between latitudes $35.0^{\circ} \mathrm{S}$ and $35.3^{\circ} \mathrm{S}$ and between longitudes $69.0^{\circ} \mathrm{W}$ and $69.4^{\circ} \mathrm{W}$ near the town of Malargüe in the province of Mendoza, Argentina, at an altitude of $1400 \mathrm{~m}$ above the sea level. The SD array, which consists of 1600 water-Cherenkov tanks $\left(10 \mathrm{~m}^{2} \times 1.2 \mathrm{~m}\right)$ spread over a triangular grid with $1500 \mathrm{~m}$ spacing, covers $3000 \mathrm{~km}^{2}$ in area [3]. The Cherenkov light emitted by the charged particles in a detector is recorded by three photo-multiplier 
tubes (PMTs). Signals are digitized with an FADC at a sampling rate of $40 \mathrm{MHz}$. The FD consists of 24 telescopes installed at four sites (six telescopes each) separated at a distance of $70 \mathrm{~km}$. Each telescope consists of a $3.5 \mathrm{~m} \times 3.5 \mathrm{~m}$ spherical mirror with a curvature radius of $3.4 \mathrm{~m}$, and a camera with a $22 \times 20$ cluster of PMTs at the focal plane. The field-of-view of a telescope is $30^{\circ}$ in elevation and $28.6^{\circ}$ in azimuth. Signals from the FD PMTs are digitized with a 10MHz-12bit FADC. Details can be found in e.g. $[1,3]$.

The TA detector site is located near Delta, Millard County, Utah, U.S., centered at $39.3^{\circ} \mathrm{N}$, $112.9^{\circ} \mathrm{W}$ at a mean altitude of $1400 \mathrm{~m}$. An array of 507 scintillation counters on a square grid with $1.2 \mathrm{~km}$ spacing covers an area of $700 \mathrm{~km}^{2}$ [4]. A counter consists of two-layers of plastic scintillators of $3 \mathrm{~m}^{2}$ area and $1.2 \mathrm{~cm}$ thick. Wavelength-shifting fibers are embedded in the scintillators, which also reduces the position dependence of the detector response in the $3 \mathrm{~m}^{2}$ area. Two PMTs are equipped for a counter, one for each layer, and signals are digitized at $50 \mathrm{MHz}$. TA FDs are installed at three sites separated with a distance of $\sim 30 \mathrm{~km}$. Technical details are given in [5].

\section{Energy measurements}

\subsection{Hybrid approach}

At a fixed energy, the expected number of particles at ground level depends significantly on the primary mass of the cosmic rays and on the hadronic interaction generator used to model the extensive air showers. By contrast, FDs provide us with an almost calorimetric measurement of the energy, except for the invisible energy carried away by muons and neutrinos. Hence, to benefit from the $\sim 100 \%$ duty cycle of the SD compared to the much lower one of the FDs that are operational on clear moon-less nights only $(\sim 12 \%)$, the energy measurement of the FD is propagated onto the SD events thanks to a subset of hybrid events detected simultaneously by both detection techniques. The SD energy estimators are built from the particle density interpolated at a pre-determined distance from the shower core, $1000 \mathrm{~m}$ for Auger and $800 \mathrm{~m}$ for TA. The Auger energy resolution is $10 \%$ at $10^{19} \mathrm{eV}$, with systematic uncertainties of $14 \%$ [6]. The TA energy resolution is $19 \%$, and systematic uncertainty is $21 \%$ [7].

\subsection{Fluorescence technique comparisons}

Although the techniques for assigning energies to events are nearly the same, there are differences as to how the final estimates are derived at the Auger Observatory and TA. A first difference is in the correction of invisible energy. A portion of the air-shower energy $(\sim 10-15 \%)$ is carried away by particles like neutrinos and muons that do not deposit all their energy in the atmosphere, and a correction is applied to the measured calorimetric energy to obtain the primary energy of the cosmic ray. In TA the invisible energy, which is a function of the cosmic-ray energy, is evaluated using Monte-Carlo simulations [9]. On the other hand, at the Auger Observatory, an empirical invisible energy estimate has been inferred using the SD data, reducing significantly the systematic uncertainties related to the high-energy hadronic interaction models and mass composition [10]. As a consequence of the deficit in the muon number in models, this estimate is considerably higher than the predictions from Monte Carlo simulations. Note that TA energies would be increased by $7 \%$ by synchronizing the invisible-energy correction to that of the Auger Observatory. 
A second notable difference in the determination of FD energy relies on the fluorescence yield, which provides the number of fluorescence photons emitted by atmospheric molecules per unit energy deposited by the charged particles in the air shower. The absolute fluorescence yield at $337 \mathrm{~nm}$ as determined by the AirFly experiment [11] with an uncertainty of $4 \%$ is used at the Auger Observatory, together with the spectrum in the $300-400 \mathrm{~nm}$ wavelength range, as well as dependencies on temperature, pressure, and humidity of the atmosphere. In TA, the measurement by Kakimoto et al. on the absolute yield [13] is used, together with the spectrum obtained in the FLASH experiment [14]. The dependencies on atmospheric density and temperature are also taken into account following [13]. Note that TA energies would decrease by $14 \%$ after synchronizing the fluorescence yield to that used at the Auger Observatory.

\subsection{Energy estimation from surface detector data}

For both SD arrays, the signal that would be detected by a station located at a reference distance from the shower axis is used as the shower-size estimator. The reference distance, $1000 \mathrm{~m}$ for Auger and $800 \mathrm{~m}$ for TA, is chosen so as to minimize the fluctuations of the shower size. The differences in reference distances stem from the detector type (water tanks, which is relatively sensitive to muons, vs scintillation counters sensitive to electrons) and the detector spacing (1500 m vs 1200 $\mathrm{m})$. To take into account atmospheric attenuation for different zenith angles of cosmic-ray arrival directions, the Auger $S(1000)$ parameter of a shower of a given zenith angle $\theta$ is converted into $S_{38}$, the particle density that would have been observed had the shower arrived at $\theta=38^{\circ}$, by means of the constant intensity cut (CIC) method [6]. The corrected shower size is subsequently calibrated against the FD energies using a power-law function, $E=A S_{38}^{B}$. The statistical uncertainty in the energy scale arising from the fit of the two calibration parameters is below 1\%. In TA, Monte-Carlo simulations of showers are used to obtain an energy "lookup-table" so as to convert $S(800)$ into primary energy for each $\theta$. Note that a CIC-based energy determination has also been carried out: for TA the energies calculated by the two methods agree within 3\% [15].

\subsection{Spectrum unfolding}

The cosmic-ray energy spectrum provides the flux of particles per steradian and per energy unit. It is primarily obtained from the observed number of events in a given energy bin divided by the exposure. However, due to effects of finite energy resolution, migration of events between neighboring energy bins occur. Even with equal migration probabilities to higher or lower energy bins, this effect can lead to significant distortions because of the steepness of the underlying spectrum. To account for it, a forward-folding method is used at the Auger Observatory, while the exposure is determined in an energy-dependent manner so as to include energy resolutions in TA.

In Auger, the differential intensity $d I / d E\left(E_{i}\right)$ is estimated as $c_{i} N_{i} /\left(\mathcal{E} \Delta E_{i}\right)$, where $N_{i}$ is the number of observed events in energy bins of width $\Delta \log _{10} E_{i}=0.1, \mathcal{E}$ is the exposure and the coefficients $c_{i}$ are the correction factors to account for resolution effects [6]. The exposure is the product of the geometrical aperture in the energy range of full efficiency and of the observation time after subtracting the dead times. The coefficients $c_{i}$ are calculated using the forward-folding approach, which consists in choosing a function $f(E ; \vec{p})$ to describe the energy spectrum with a set of parameters $\vec{p}$ that include the spectral indices, the break energies, the width transitions and the overall scaling factor. The function is subsequently folded for detector effects and used to 
estimate the expected number of events $\mu_{i}(\vec{p})$. The set of parameters $\vec{p}$ that best describe the data is determined through a likelihood procedure comparing $\mu_{i}(\vec{p})$ and the measurements $N_{i}$. Finally the correction coefficients are determined through the ratio $c_{i}(\vec{p})=\mu_{i}^{\prime}(\vec{p}) / \mu_{i}(\vec{p})$, where $\mu_{i}^{\prime}$ is the expected number of events without detector effects. The coefficients are found to be close to 1 over the whole energy range with a mild energy dependence. Overall, by characterizing the SD response with the measurements performed with the hybrid events, the spectrum obtained is independent of model and primary mass assumptions [6].

The TA SD array is not fully efficient below $10^{19} \mathrm{eV}$, and the aperture and exposure $\mathcal{E}(E)$ are evaluated by Monte-Carlo simulations as a function of reconstructed energy $E$, assuming an underlying energy spectrum $\left(\sim E^{-3}\right)$. The bin-to-bin migration effect is encoded in $\mathcal{E}(E)$, which is therefore not constant even in the energy region of $100 \%$ trigger efficiency. The differential intensity $d I / d E\left(E_{i}\right)$ is then calculated as $N_{i} / \mathcal{E}\left(E_{i}\right)$. The spectrum fit to a function $f(E ; \vec{p})$ is done by comparing the observed number of events $N_{i}$ and the expected numbers $\mu_{i}(\vec{p})=\mathcal{E}\left(E_{i}\right) f\left(E_{i} ; \vec{p}\right)$ assuming Poisson statistics.

\section{The energy spectrum of ultra-high energy cosmic rays}

The Auger and TA energy spectra are presented in Figure $1[6,7]$. Beyond the well-established "ankle", a hardening of the spectrum at $E \sim 5 \times 10^{18} \mathrm{eV}$ where the spectral index $d I / d E \propto E^{-\gamma}$ changes from $\gamma=3.2-3.3$ to $\gamma=2.6-2.7$, the two spectra have also captured a steepening at around $E \sim 5 \times 10^{19} \mathrm{eV}$, above which the cosmic-ray intensity drastically falls off with $\gamma \sim 5$. The spectral shape and the position of the steepening in the TA spectrum can be fit by a "GZK scenario" in which a pure-proton composition is assumed. On the other hand, the Auger spectrum and composition data are suggestive of cosmic rays getting heavier with energy. In this scenario, the steepening is caused by both the GZK effect and the maximal acceleration energy at the sources close to $10^{20} \mathrm{eV}$ [8]. The origin of the high-energy steepening is currently one of the most important problems in cosmic-ray physics.

There is a systematic difference in the absolute energy scale between the two measurements at a level of $\sim 9 \%$. If we rescale the energies by $+4.5 \%$ for Auger and $-4.5 \%$ for TA, values well within the systematic uncertainties of both experiments, a better agreement of the spectra is seen as shown in the right panel of Figure 1. It is worth noting that the overall energy scale offset of $9 \%$ is significantly reduced once the differences in the energy assignments arising from the fluorescence yield and invisible energy models adopted by the two Collaborations are subtracted for (see Sec. $3.2)$.

\subsection{Comparisons in common declination band}

When we compare, after the $\pm 4.5 \%$ rescalings, the energy spectra in the declination band that is commonly accessible to the two observatories $\left(-15^{\circ} \leq \delta \leq+24.8^{\circ}\right)$, the differences are smaller (left panel of Figure 2). However, the persistent differences require an additional energy rescaling in an energy-dependent way $\left( \pm 10 \% /\right.$ decade for $\left.E>10^{19} \mathrm{eV}\right)$ to get agreement (right panel of Figure 2). A Monte-Carlo study is underway to disentangle systematic from statistical effects. The statistical significance of the energy-dependent energy shift obtained using an enlarged data set that 

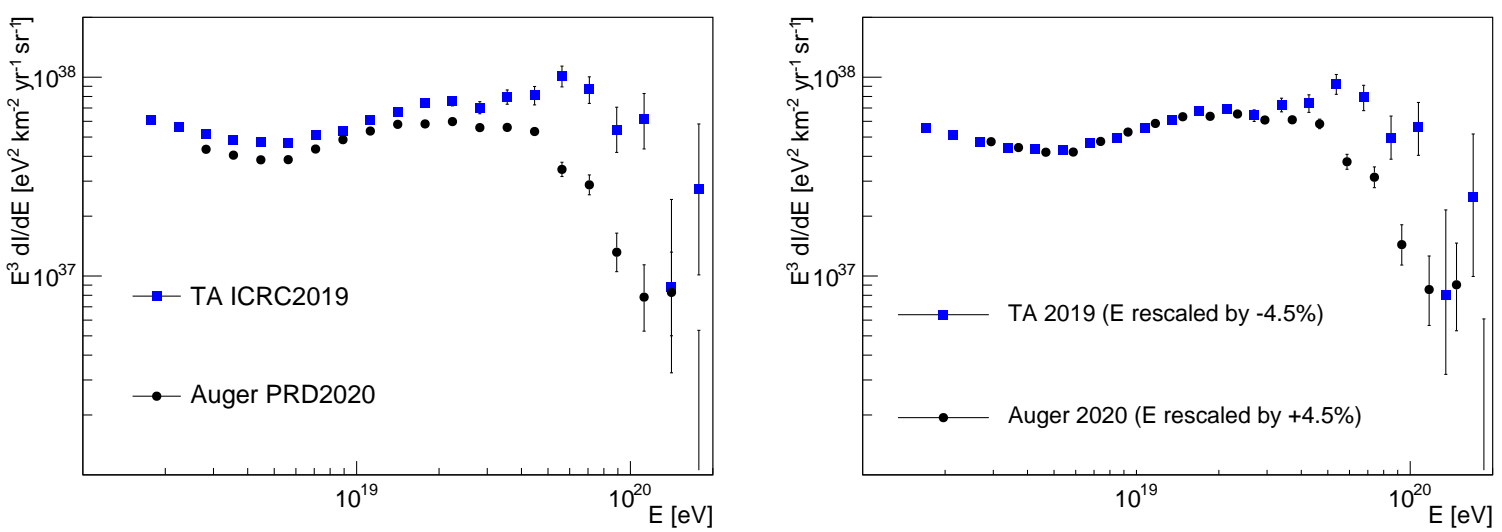

Figure 1: Left: Auger and TA energy spectra in the full fields of view $\left(-90^{\circ}<\delta<+24.8^{\circ}\right.$ and $-15.7^{\circ}<\delta<$ $+90^{\circ}$, respectively). Right: energy-rescaled spectra by the same amount $( \pm 4.5 \%)$ and in opposite directions.
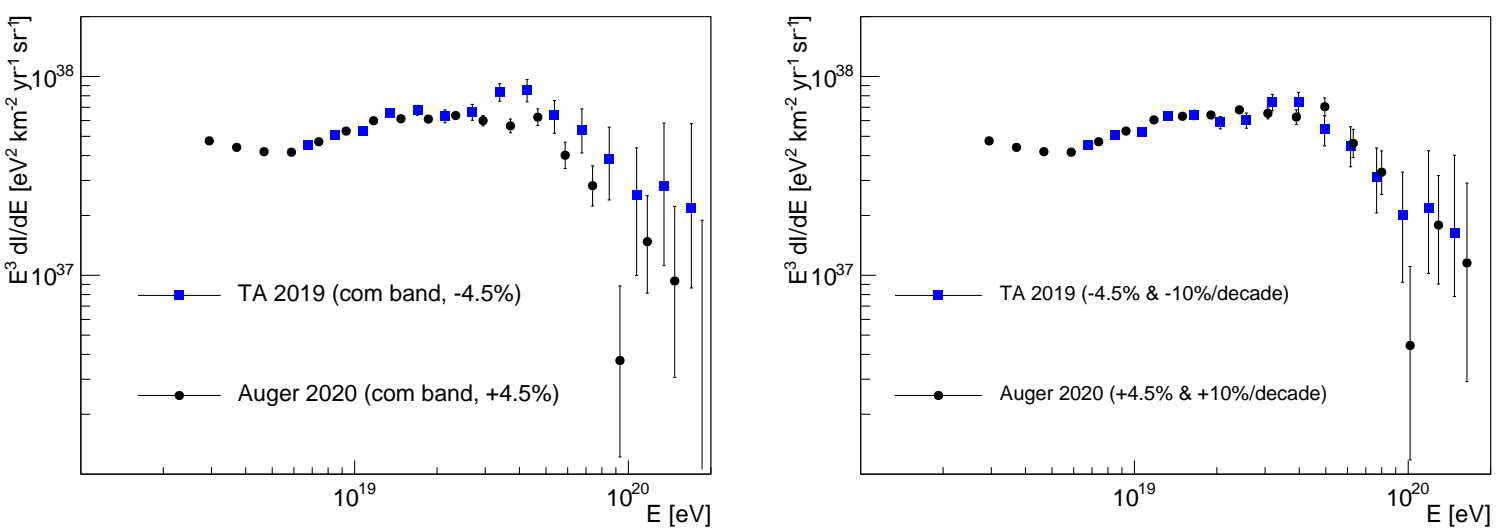

Figure 2: Left: Auger and TA spectra in the common declination band $\left(-15.7^{\circ}<\delta<24.8^{\circ}\right)$ with a constant shift $\pm 4.5 \%$, Right: with an energy-dependent shift $\pm 10 \% \times \log _{10}\left(E / 10^{19} \mathrm{eV}\right)$ for $E>10^{19} \mathrm{eV}$.

extends up to higher declinations (up to $+44.8^{\circ}$ including the Auger events of large zenith angles) is $3.7 \sigma$ [18].

The Auger spectra in different declination bands are fully consistent within the accessible field-of-view $[6,8]$. On the other hand, TA observed slightly different spectra in the northern and the southern part of the TA sky with different positions of the steepening at a $3.5 \sigma$ confidence level [19]. No systematic and instrumental effects have been identified, and the difference remains after removing events of the TA "hotspot" located at $(\alpha, \delta)=\left(146.7^{\circ}, 43.2^{\circ}\right)$ with a $20^{\circ}$ radius [20].

\subsection{New feature in the spectral shape}

A new feature in the Auger spectrum of cosmic rays above $10^{19} \mathrm{eV}$ has been reported [6]. With the "instep" feature, the steepening is no longer modeled with a simple break, as there is another one prior to the high energy fall-off. The Auger spectrum hence exhibits three breaks at 


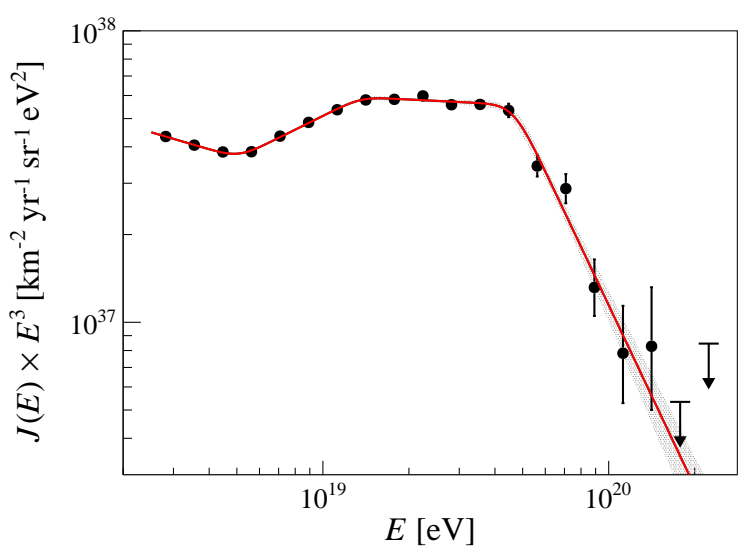

\begin{tabular}{ccc} 
Parameter & Auger & TA \\
\hline$\gamma_{1}$ & $3.29 \pm 0.02$ & $3.23 \pm 0.01$ \\
$\gamma_{2}$ & $2.51 \pm 0.03$ & $2.63 \pm 0.02$ \\
$\gamma_{3}$ & $3.05 \pm 0.05$ & $2.92 \pm 0.06$ \\
$\gamma_{4}$ & $5.1 \pm 0.3$ & $5.0 \pm 0.4$ \\
$E_{\text {ankle }} / E e V$ & $5.0 \pm 0.1$ & $5.4 \pm 0.1$ \\
$E_{\text {instep }} / E e V$ & $13 \pm 1$ & $18 \pm 1$ \\
$E_{\text {cut }} / E e V$ & $46 \pm 3$ & $71 \pm 3$
\end{tabular}

Figure 3: The Auger energy spectrum fitted with four power-laws[6], and the fit parameters (three break points $E_{\text {ankle }}, E_{\text {instep }}, E_{\text {cut }}$ and four power-law indices). The errors quoted are the statistical errors. The systematic uncertainties on the break points are $\Delta \log _{10} E=0.06$ for Auger and 0.09 for TA.

$E=10^{18.70} \mathrm{eV}, 10^{19.11} \mathrm{eV}$, and $10^{19.66} \mathrm{eV}$, with changes of spectral indices $\gamma=3.29 \rightarrow 2.51 \rightarrow$ $3.05 \rightarrow$ 5.1, as presented in Figure 3 .

For over a decade the Auger Collaboration characterized the energy spectrum with a function in which the spectral index between the ankle and the suppression at highest energies evolves slowly from $\approx 2.5$ to $\approx 5$. The statistical significance with which this behaviour has been discarded in favour of the introduction of the new intermediate break is $3.9 \sigma$. No dependence on horizontal coordinates nor equatorial ones were found, other than a mild excess from the Southern Hemisphere consistent with an expectation from the reported dipole anisotropy [16]. The spectrum, including the newlyreported structure, can be reproduced by a model with an energy-dependent mass composition [8].

A similar feature of two-step softening of the spectrum has also been identified in the northern hemisphere by using HiRes and TA data [19]. Characterizing the spectrum between the ankle energy and $E=10^{19.25} \mathrm{eV}$, the spectral index is found to be $\gamma=2.63$. The number of events expected between $E=10^{19.25 ~ 19.85} \mathrm{eV}$ if this power law were to hold above $E=10^{19.25} \mathrm{eV}$ would be 1269 , while 1086 events are observed. The single power-law extrapolation is thus rejected with $5.3 \sigma$ confidence. The previously-reported break at $10^{19.85} \mathrm{eV}$ still holds. Overall, a series of power laws describes the TA spectrum, with $\gamma=3.23 \rightarrow 2.63 \rightarrow 2.92 \rightarrow 5.0$. The spectral fitting parameters to a series of power laws are listed in Figure 3. Composition studies of the northern hemisphere data are crucial to interpret the fine structure of the spectrum in the highest energy region.

\section{Summary}

We have reviewed the present status of measurements of the cosmic-ray energy spectrum at the highest energies at the Pierre Auger Observatory and the Telescope Array. The spectra measured by the two experiments are basically in agreement in the energy range $E<10^{19} \mathrm{eV}$, 
except for a difference in the absolute energy scale at a level of $9 \%$, which is well within the systematic uncertainties. An even better agreement has been seen in the common declination band $-15^{\circ} \leq \delta \leq 24.8^{\circ}$. However a difference still persists at the highest energies. Besides, no indication of declination dependence is found in Auger, while TA data suggest different steepening positions for events below and above $\delta=24.8^{\circ}$, which may indicate a different energy spectrum of cosmic rays in the northern hemisphere. We also discussed a new "instep" feature in the fine structure of the spectrum: the high-energy steepening is not a simple break, but a combination of a softening at $10^{19.11-19.26} \mathrm{eV}$ from $\gamma \sim 2.5$ to $\gamma \sim 3$ and a steepening at $10^{19.66-19.85}$ to $\gamma \sim 5$. First observed at the Auger Observatory, it has been subsequently supported by the northern-hemisphere data from HiRes and TA. No systematic effects have been found, and this feature must be of astrophysical origin. The features of the Auger spectrum can be reproduced with models injecting a mass composition of cosmic rays getting heavier with energy. This is in line with the mass composition inferred from Auger data on depths of shower maximum, while larger statistics is needed in the northern hemisphere to probe the presence of helium or heavier elements in this energy region.

Both in Auger and TA, upgrades of the detectors are ongoing. These are the so-called AugerPrime and TA $\times 4$. AugerPrime employs scintillation counters on top of the existing waterCherenkov tanks, to enhance the sensitivity to the electromagnetic components of the air showers and hence on mass-discrimination power. TA $\times 4$ quadruples the effective area of the original TA SD array installing additional 250 scintillation counters in the northern and southern "lobes". Further data analysis with increased statistics and composition measurements will permit to disentangle the differences in the two experiments and interpretation both in technical and astrophysical aspects.

\section{References}

[1] V. Verzi, D. Ivanov and Y. Tsunesada, Prog. Theor. Exp. Phys., 12A103 (2017)

[2] O. Deligny et al.,The Auger-TA joint working group report, Proceedings of ICRC2019 (2019)

[3] The Pierre Auger Collaboration, Nucl. Instr. Meth. A, 798, 172 (2015)

[4] The Telescope Array Collaboration, Nucl. Instr. Meth. A, 689, 187 (2013)

[5] The Telescope Array Collaboration, Nucl. Instr. Meth. A, 676, 54 (2012)

[6] The Pierre Auger Collaboration, Phys. Rev. D, 102, 062005 (2020)

[7] D. Ivanov, Proceedings of ICRC2019 (2019)

[8] The Pierre Auger Collaboration, Phys. Rev. Lett., 125, 121106 (2020)

[9] Y. Tsunesada, Proceeding of ICRC2011 Beijing (2011)

[10] The Pierre Auger Collaboration, Phys. Rev. D, 100 (2019) 082003

[11] AIRFLY Collaboration, Astropart. Phys., 42, 90 (2013).

[12] AIRFLY Collaboration, Astropart. Phys., 28, 41 (2007).

[13] F. Kakimoto et al., Nucl. Instrum. Methods A, 372, 527 (1996).

[14] R. Abbasi et al., Astropart. Phys., 29, 77 (2008)

[15] D. Ivanov, UHECR2018 Proceedings (2018)

[16] The Pierre Auger Collaboration, Astrophys. J., 868, 4 (2018)

[17] T. Abu-Zayyad et al. (Telescope Array and Pierre Auger Collaborations), EPJ Web Conf. 210, 01002 (2019).

[18] P. Tinyakov et al. (Telescope Array and Pierre Auger Collaborations), these proceedings.

[19] D. Ivanov for the Telescope Array Collaboration, this conference contribution.

[20] The Telescope Array Collaboration, Astrophys. J. Lett., 790, L21 (2014) 


\section{The Pierre Auger Collaboration}

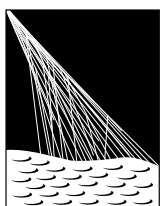

PIERRE

P. Abreu ${ }^{72}$, M. Aglietta ${ }^{54,52}$, J.M. Albury ${ }^{13}$, I. Allekotte ${ }^{1}$, A. Almela ${ }^{8,12}$, J. AlvarezMuñiz ${ }^{79}$, R. Alves Batista ${ }^{80}$, G.A. Anastasi ${ }^{63,52}$, L. Anchordoqui ${ }^{87}$, B. Andrada ${ }^{8}$, S. Andringa ${ }^{72}$, C. Aramo ${ }^{50}$, P.R. Araújo Ferreira ${ }^{42}$, J. C. Arteaga Velázquez ${ }^{67}$, H. Asorey ${ }^{8}$, P. Assis ${ }^{72}$, G. Avila ${ }^{11}$, A.M. Badescu ${ }^{75}$, A. Bakalova ${ }^{32}$, A. Balaceanu ${ }^{73}$, F. Barbato ${ }^{45,46}$, R.J. Barreira Luz ${ }^{72}$, K.H. Becker ${ }^{38}$, J.A. Bellido ${ }^{13,69}$, C. Berat ${ }^{36}$, M.E. Bertaina ${ }^{63,52}$, X. Bertou ${ }^{1}$, P.L. Biermann ${ }^{b}$, V. Binet ${ }^{6}$, K. Bismark ${ }^{39,8}$, T. Bister ${ }^{42}$, J. Biteau ${ }^{37}$, J. Blazek ${ }^{32}$, C. Bleve ${ }^{36}$, M. Boháčová ${ }^{32}$, D. Boncioli ${ }^{57,46}$, C. Bonifazi ${ }^{9,26}$, L. Bonneau Arbeletche ${ }^{21}$, N. Borodai ${ }^{70}$, A.M. Botti ${ }^{8}$, J. Brack $^{d}$, T. Bretz ${ }^{42}$, P.G. Brichetto Orchera ${ }^{8}$, F.L. Briechle ${ }^{42}$, P. Buchholz ${ }^{44}$, A. Bueno ${ }^{78}$, S. Buitink ${ }^{15}$, M. Buscemi ${ }^{47}$, M. Büsken ${ }^{39,8}$, K.S. Caballero-Mora ${ }^{66}$, L. Caccianiga ${ }^{59,49}$, F. Canfora ${ }^{80,81}$, I. Caracas ${ }^{38}$, J.M. Carceller ${ }^{78}$, R. Caruso ${ }^{58,47}$, A. Castellina ${ }^{54,52}$, F. Catalani ${ }^{19}$, G. Cataldi ${ }^{48}$, L. Cazon $^{72}$, M. Cerda ${ }^{10}$, J.A. Chinellato ${ }^{22}$, J. Chudoba ${ }^{32}$, L. Chytka ${ }^{33}$, R.W. Clay ${ }^{13}$, A.C. Cobos Cerutti ${ }^{7}$, R. Colalillo ${ }^{60,50}$, A. Coleman ${ }^{93}$, M.R. Coluccia ${ }^{48}$, R. Conceição ${ }^{72}$, A. Condorelli ${ }^{45,46}$, G. Consolati ${ }^{49,55}$, F. Contreras ${ }^{11}$, F. Convenga ${ }^{56,48}$, D. Correia dos Santos ${ }^{28}$, C.E. Covault ${ }^{85}$, S. Dasso ${ }^{5,3}$, K. Daumiller ${ }^{41}$, B.R. Dawson ${ }^{13}$, J.A. Day ${ }^{13}$, R.M. de Almeida ${ }^{28}$, J. de Jesús ${ }^{8,41}$, S.J. de Jong ${ }^{80,81}$, G. De Mauro ${ }^{80,81}$, J.R.T. de Mello Neto ${ }^{26,27}$, I. De Mitri ${ }^{45,46}$, J. de Oliveira ${ }^{18}$, D. de Oliveira Franco ${ }^{22}$, F. de Palma ${ }^{56,48}$, V. de Souza $^{20}$, E. De Vito ${ }^{56,48}$, M. del Río ${ }^{11}$, O. Deligny ${ }^{34}$, L. Deval ${ }^{41,8}$, A. di Matteo $^{52}$, C. Dobrigkeit ${ }^{22}$, J.C. D’Olivo ${ }^{68}$, L.M. Domingues Mendes ${ }^{72}$, R.C. dos Anjos ${ }^{25}$, D. dos Santos ${ }^{28}$, M.T. Dova ${ }^{4}$, J. Ebr ${ }^{32}$, R. Engel ${ }^{39,41}$, I. Epicoco ${ }^{56,48}$, M. Erdmann ${ }^{42}$, C.O. Escobar ${ }^{a}$, A. Etchegoyen ${ }^{8,12}$, H. Falcke $80,82,81$, J. Farmer ${ }^{92}$, G. Farrar ${ }^{90}$, A.C. Fauth ${ }^{22}$, N. Fazzini ${ }^{a}$, F. Feldbusch ${ }^{40}$, F. Fenu ${ }^{54,52}$, B. Fick ${ }^{89}$, J.M. Figueira ${ }^{8}$, A. Filipčič ${ }^{77,76}$, T. Fitoussi ${ }^{41}$, T. Fodran ${ }^{80}$, M.M. Freire ${ }^{6}$, T. Fujii ${ }^{92, e}$, A. Fuster ${ }^{8,12}$, C. Galea ${ }^{80}$, C. Galelli ${ }^{59,49}$, B. García ${ }^{7}$, A.L. Garcia Vegas ${ }^{42}$, H. Gemmeke ${ }^{40}$, F. Gesualdi ${ }^{8,41}$, A. Gherghel-Lascu ${ }^{73}$, P.L. Ghia ${ }^{34}$, U. Giaccari ${ }^{80}$, M. Giammarchi ${ }^{49}$, J. Glombitza ${ }^{42}$, F. Gobbi ${ }^{10}$, F. Gollan ${ }^{8}$, G. Golup ${ }^{1}$, M. Gómez Berisso ${ }^{1}$, P.F. Gómez Vitale ${ }^{11}$, J.P. Gongora ${ }^{11}$, J.M. González ${ }^{1}$, N. González ${ }^{14}$, I. Goos ${ }^{1,41}$, D. Góra ${ }^{70}$, A. Gorgi ${ }^{54,52}$, M. Gottowik ${ }^{38}$, T.D. Grubb ${ }^{13}$, F. Guarino ${ }^{60,50}$, G.P. Guedes ${ }^{23}$, E. Guido ${ }^{52,63}$, S. Hahn ${ }^{41,8}$, P. Hamal $^{32}$, M.R. Hampel ${ }^{8}$, P. Hansen ${ }^{4}$, D. Harari ${ }^{1}$, V.M. Harvey ${ }^{13}$, A. Haungs ${ }^{41}$, T. Hebbeker ${ }^{42}$, D. Heck ${ }^{41}$, G.C. Hill ${ }^{13}$, C. Hojvat ${ }^{a}$, J.R. Hörandel ${ }^{80,81}$, P. Horvath ${ }^{33}$, M. Hrabovský ${ }^{33}$, T. Huege ${ }^{41,15}$, A. Insolia ${ }^{58,47}$, P.G. Isar ${ }^{74}$, P. Janecek ${ }^{32}$, J.A. Johnsen ${ }^{86}$, J. Jurysek ${ }^{32}$, A. Kääpä ${ }^{38}$, K.H. Kampert ${ }^{38}$, N. Karastathis ${ }^{41}$, B. Keilhauer ${ }^{41}$, J. Kemp ${ }^{42}$, A. Khakurdikar ${ }^{80}$, V.V. Kizakke Covilakam $^{8,41}$, H.O. Klages ${ }^{41}$, M. Kleifges ${ }^{40}$, J. Kleinfeller ${ }^{10}$, M. Köpke ${ }^{39}$, N. Kunka ${ }^{40}$, B.L. Lago ${ }^{17}$, R.G. Lang ${ }^{20}$, N. Langner ${ }^{42}$, M.A. Leigui de Oliveira ${ }^{24}$, V. Lenok ${ }^{41}$, A. Letessier-Selvon ${ }^{35}$, I. LhenryYvon $^{34}$, D. Lo Presti ${ }^{58,47}$, L. Lopes ${ }^{72}$, R. López ${ }^{64}$, L. Lu ${ }^{94}$, Q. Luce ${ }^{39}$, J.P. Lundquist ${ }^{76}$, A. Machado Payeras $^{22}$, G. Mancarella ${ }^{56,48}$, D. Mandat ${ }^{32}$, B.C. Manning ${ }^{13}$, J. Manshanden $^{43}$, P. Mantsch $^{a}$, S. Marafico $^{34}$, A.G. Mariazzi ${ }^{4}$, I.C. Mariş ${ }^{14}$, G. Marsella ${ }^{61,47}$, D. Martello ${ }^{56,48}$, S. Martinellii ${ }^{41,8}$, O. Martínez Bravo ${ }^{64}$, M. Mastrodicasa ${ }^{57,46}$, H.J. Mathes ${ }^{41}$, J. Matthews ${ }^{88}$, G. Matthiae ${ }^{62,51}$, E. Mayotte ${ }^{38}$, P.O. Mazur ${ }^{a}$, G. MedinaTanco $^{68}$, D. Melo ${ }^{8}$, A. Menshikov ${ }^{40}$, K.-D. Merenda ${ }^{86}$, S. Michal ${ }^{33}$, M.I. Micheletti ${ }^{6}$, L. Miramonti ${ }^{59,49}$, S. Mollerach ${ }^{1}$, F. Montanet ${ }^{36}$, C. Morello ${ }^{54,52}$, M. Mostafá ${ }^{91}$, A.L. Müller ${ }^{8}$, M.A. Muller ${ }^{22}$, K. Mulrey ${ }^{15}$, R. Mussa ${ }^{52}$, M. Muzio ${ }^{90}$, W.M. Namasaka ${ }^{38}$, A. Nasr-Esfahani ${ }^{38}$, L. Nellen ${ }^{68}$, M. Niculescu-Oglinzanu ${ }^{73}$, M. Niechciol ${ }^{44}$, D. Nitz ${ }^{89}$, D. Nosek ${ }^{31}$, V. Novotny ${ }^{31}$, L. Nožka $^{33}$, A Nucita $^{56,48}$, L.A. Núñez ${ }^{30}$, M. Palatka ${ }^{32}$, J. Pallotta ${ }^{2}$, P. Papenbreer ${ }^{38}$, G. Parente ${ }^{79}$, A. Parra ${ }^{64}$, J. Pawlowsky ${ }^{38}$, M. Pech ${ }^{32}$, F. Pedreira ${ }^{79}$, J. Pȩkala ${ }^{70}$, R. Pelayo ${ }^{65}$, J. Peña-Rodriguez ${ }^{30}$, E.E. Pereira Martins ${ }^{39,8}$, J. Perez Armand ${ }^{21}$, C. Pérez Bertolli $^{8,41}$, M. Perlin ${ }^{8,41}$, L. Perrone ${ }^{56,48}$, S. Petrera ${ }^{45,46}$, T. Pierog ${ }^{41}$, M. Pimenta ${ }^{72}$, V. Pirronello ${ }^{58,47}$, M. Platino ${ }^{8}$, B. Pont ${ }^{80}$, M. Pothast ${ }^{81,80}$, P. Privitera ${ }^{92}$, M. Prouza ${ }^{32}$, A. Puyleart ${ }^{89}$, S. Querchfeld ${ }^{38}$, J. Rautenberg ${ }^{38}$, D. Ravignani ${ }^{8}$, M. Reininghaus ${ }^{41,8}$, J. Ridky ${ }^{32}$, F. Riehn ${ }^{72}$, M. Risse ${ }^{44}$, V. Rizi ${ }^{57,46}$, W. Rodrigues de Carvalho ${ }^{21}$, J. Rodriguez Rojo ${ }^{11}$, M.J. Roncoroni ${ }^{8}$, S. Rossoni ${ }^{43}$, M. Roth ${ }^{41}$, E. Roulet ${ }^{1}$, A.C. Rovero ${ }^{5}$, P. Ruehl ${ }^{44}$, A. Saftoiu ${ }^{73}$, F. Salamida ${ }^{57,46}$, H. Salazar ${ }^{64}$, G. Salina ${ }^{51}$, J.D. Sanabria Gomez ${ }^{30}$, 
F. Sánchez ${ }^{8}$, E.M. $\operatorname{Santos}^{21}$, E. $\operatorname{Santos}^{32}$, F. Sarazin ${ }^{86}$, R. Sarmento ${ }^{72}$, C. Sarmiento-Cano ${ }^{8}$, R. Sato ${ }^{11}$, P. Savina ${ }^{56,48,34,94}$, C.M. Schäfer ${ }^{41}$, V. Scherini ${ }^{56,48}$, H. Schieler ${ }^{41}$, M. Schimassek ${ }^{39,8}$, M. Schimp ${ }^{38}$, F. Schlüter ${ }^{41,8}$, D. Schmidt ${ }^{39}$, O. Scholten ${ }^{84,15}$, P. Schovánek ${ }^{32}$, F.G. Schröder ${ }^{93,41}$, S. Schröder ${ }^{38}$, J. Schulte ${ }^{42}$, S.J. Sciutto ${ }^{4}$, M. Scornavacche ${ }^{8,41}$, A. Segreto ${ }^{53,47}$, S. Sehgal ${ }^{38}$, R.C. Shellard ${ }^{16}$, G. Sigl ${ }^{43}$, G. Silli ${ }^{8,41}$, O. Sima ${ }^{73, f}$, R. Šmída ${ }^{92}$, P. Sommers ${ }^{91}$, J.F. Soriano ${ }^{87}$, J. Souchard ${ }^{36}$, R. Squartini ${ }^{10}$, M. Stadelmaier ${ }^{41,8}$, D. Stanca ${ }^{73}$, S. Stanič ${ }^{76}$, J. Stasielak ${ }^{70}$, P. Stassi ${ }^{36}$, A. Streich ${ }^{39,8}$, M. Suárez-Durán ${ }^{14}$, T. Sudholz ${ }^{13}$, T. Suomijärvi ${ }^{37}$, A.D. Supanitsky ${ }^{8}$, Z. Szadkowski ${ }^{71}$, A. Tapia ${ }^{29}$, C. Taricco ${ }^{63,52}$, C. Timmermans ${ }^{81,80}$, O. Tkachenko ${ }^{41}$, P. Tobiska ${ }^{32}$, C.J. Todero Peixoto ${ }^{19}$, B. Tomé ${ }^{72}$, Z. Torrès ${ }^{36}$, A. Travaini ${ }^{10}$, P. Travnicek ${ }^{32}$, C. Trimarelli ${ }^{57,46}$, M. Tueros ${ }^{4}$, R. Ulrich ${ }^{41}$, M. Unger ${ }^{41}$, L. Vaclavek ${ }^{33}$, M. Vacula ${ }^{33}$, J.F. Valdés Galicia ${ }^{68}$, L. Valore ${ }^{60,50}$, E. Varela ${ }^{64}$, A. Vásquez-Ramírez ${ }^{30}$, D. Veberič ${ }^{41}$, C. Ventura ${ }^{27}$, I.D. Vergara Quispe ${ }^{4}$, V. Verzi ${ }^{51}$, J. Vicha ${ }^{32}$, J. Vink ${ }^{83}$, S. Vorobiov ${ }^{76}$, H. Wahlberg ${ }^{4}$, C. Watanabe ${ }^{26}$, A.A. Watson ${ }^{c}$, M. Weber $^{40}$, A. Weindl ${ }^{41}$, L. Wiencke ${ }^{86}$, H. Wilczyński ${ }^{70}$, M. Wirtz ${ }^{42}$, D. Wittkowski ${ }^{38}$, B. Wundheiler ${ }^{8}$, A. Yushkov $^{32}$, O. Zapparrata ${ }^{14}$, E. Zas ${ }^{79}$, D. Zavrtanik ${ }^{76,77}$, M. Zavrtanik ${ }^{77,76}$, L. Zehrer ${ }^{76}$

${ }^{1}$ Centro Atómico Bariloche and Instituto Balseiro (CNEA-UNCuyo-CONICET), San Carlos de Bariloche, Argentina

${ }^{2}$ Centro de Investigaciones en Láseres y Aplicaciones, CITEDEF and CONICET, Villa Martelli, Argentina

${ }^{3}$ Departamento de Física and Departamento de Ciencias de la Atmósfera y los Océanos, FCEyN, Universidad de Buenos Aires and CONICET, Buenos Aires, Argentina

${ }^{4}$ IFLP, Universidad Nacional de La Plata and CONICET, La Plata, Argentina

${ }^{5}$ Instituto de Astronomía y Física del Espacio (IAFE, CONICET-UBA), Buenos Aires, Argentina

${ }^{6}$ Instituto de Física de Rosario (IFIR) - CONICET/U.N.R. and Facultad de Ciencias Bioquímicas y Farmacéuticas U.N.R., Rosario, Argentina

${ }^{7}$ Instituto de Tecnologías en Detección y Astropartículas (CNEA, CONICET, UNSAM), and Universidad Tecnológica Nacional - Facultad Regional Mendoza (CONICET/CNEA), Mendoza, Argentina

${ }^{8}$ Instituto de Tecnologías en Detección y Astropartículas (CNEA, CONICET, UNSAM), Buenos Aires, Argentina

${ }^{9}$ International Center of Advanced Studies and Instituto de Ciencias Físicas, ECyT-UNSAM and CONICET, Campus Miguelete - San Martín, Buenos Aires, Argentina

${ }^{10}$ Observatorio Pierre Auger, Malargüe, Argentina

${ }^{11}$ Observatorio Pierre Auger and Comisión Nacional de Energía Atómica, Malargüe, Argentina

12 Universidad Tecnológica Nacional - Facultad Regional Buenos Aires, Buenos Aires, Argentina

${ }^{13}$ University of Adelaide, Adelaide, S.A., Australia

${ }^{14}$ Université Libre de Bruxelles (ULB), Brussels, Belgium

15 Vrije Universiteit Brussels, Brussels, Belgium

${ }^{16}$ Centro Brasileiro de Pesquisas Fisicas, Rio de Janeiro, RJ, Brazil

${ }^{17}$ Centro Federal de Educação Tecnológica Celso Suckow da Fonseca, Nova Friburgo, Brazil

18 Instituto Federal de Educação, Ciência e Tecnologia do Rio de Janeiro (IFRJ), Brazil

${ }^{19}$ Universidade de São Paulo, Escola de Engenharia de Lorena, Lorena, SP, Brazil

${ }^{20}$ Universidade de São Paulo, Instituto de Física de São Carlos, São Carlos, SP, Brazil

${ }^{21}$ Universidade de São Paulo, Instituto de Física, São Paulo, SP, Brazil

22 Universidade Estadual de Campinas, IFGW, Campinas, SP, Brazil

${ }^{23}$ Universidade Estadual de Feira de Santana, Feira de Santana, Brazil

${ }^{24}$ Universidade Federal do ABC, Santo André, SP, Brazil

${ }^{25}$ Universidade Federal do Paraná, Setor Palotina, Palotina, Brazil

${ }^{26}$ Universidade Federal do Rio de Janeiro, Instituto de Física, Rio de Janeiro, RJ, Brazil

${ }^{27}$ Universidade Federal do Rio de Janeiro (UFRJ), Observatório do Valongo, Rio de Janeiro, RJ, Brazil

${ }^{28}$ Universidade Federal Fluminense, EEIMVR, Volta Redonda, RJ, Brazil

${ }^{29}$ Universidad de Medellín, Medellín, Colombia

${ }^{30}$ Universidad Industrial de Santander, Bucaramanga, Colombia

${ }^{31}$ Charles University, Faculty of Mathematics and Physics, Institute of Particle and Nuclear Physics, Prague, Czech Republic 
${ }^{32}$ Institute of Physics of the Czech Academy of Sciences, Prague, Czech Republic

33 Palacky University, RCPTM, Olomouc, Czech Republic

${ }^{34}$ CNRS/IN2P3, IJCLab, Université Paris-Saclay, Orsay, France

${ }^{35}$ Laboratoire de Physique Nucléaire et de Hautes Energies (LPNHE), Sorbonne Université, Université de Paris, CNRSIN2P3, Paris, France

${ }^{36}$ Univ. Grenoble Alpes, CNRS, Grenoble Institute of Engineering Univ. Grenoble Alpes, LPSC-IN2P3, 38000 Grenoble, France

${ }^{37}$ Université Paris-Saclay, CNRS/IN2P3, IJCLab, Orsay, France

38 Bergische Universität Wuppertal, Department of Physics, Wuppertal, Germany

${ }^{39}$ Karlsruhe Institute of Technology (KIT), Institute for Experimental Particle Physics, Karlsruhe, Germany

${ }^{40}$ Karlsruhe Institute of Technology (KIT), Institut für Prozessdatenverarbeitung und Elektronik, Karlsruhe, Germany

${ }^{41}$ Karlsruhe Institute of Technology (KIT), Institute for Astroparticle Physics, Karlsruhe, Germany

42 RWTH Aachen University, III. Physikalisches Institut A, Aachen, Germany

${ }^{43}$ Universität Hamburg, II. Institut für Theoretische Physik, Hamburg, Germany

${ }^{44}$ Universität Siegen, Department Physik - Experimentelle Teilchenphysik, Siegen, Germany

${ }^{45}$ Gran Sasso Science Institute, L'Aquila, Italy

${ }^{46}$ INFN Laboratori Nazionali del Gran Sasso, Assergi (L'Aquila), Italy

${ }^{47}$ INFN, Sezione di Catania, Catania, Italy

${ }^{48}$ INFN, Sezione di Lecce, Lecce, Italy

${ }^{49}$ INFN, Sezione di Milano, Milano, Italy

50 INFN, Sezione di Napoli, Napoli, Italy

51 INFN, Sezione di Roma “Tor Vergata”, Roma, Italy

52 INFN, Sezione di Torino, Torino, Italy

${ }^{53}$ Istituto di Astrofisica Spaziale e Fisica Cosmica di Palermo (INAF), Palermo, Italy

54 Osservatorio Astrofisico di Torino (INAF), Torino, Italy

55 Politecnico di Milano, Dipartimento di Scienze e Tecnologie Aerospaziali , Milano, Italy

56 Università del Salento, Dipartimento di Matematica e Fisica "E. De Giorgi”, Lecce, Italy

57 Università dell'Aquila, Dipartimento di Scienze Fisiche e Chimiche, L'Aquila, Italy

58 Università di Catania, Dipartimento di Fisica e Astronomia, Catania, Italy

${ }^{59}$ Università di Milano, Dipartimento di Fisica, Milano, Italy

${ }^{60}$ Università di Napoli "Federico II", Dipartimento di Fisica "Ettore Pancini”, Napoli, Italy

${ }^{61}$ Università di Palermo, Dipartimento di Fisica e Chimica "E. Segrè", Palermo, Italy

62 Università di Roma “Tor Vergata”, Dipartimento di Fisica, Roma, Italy

63 Università Torino, Dipartimento di Fisica, Torino, Italy

64 Benemérita Universidad Autónoma de Puebla, Puebla, México

${ }^{65}$ Unidad Profesional Interdisciplinaria en Ingeniería y Tecnologías Avanzadas del Instituto Politécnico Nacional (UPIITA-IPN), México, D.F., México

66 Universidad Autónoma de Chiapas, Tuxtla Gutiérrez, Chiapas, México

${ }^{67}$ Universidad Michoacana de San Nicolás de Hidalgo, Morelia, Michoacán, México

${ }^{68}$ Universidad Nacional Autónoma de México, México, D.F., México

${ }^{69}$ Universidad Nacional de San Agustin de Arequipa, Facultad de Ciencias Naturales y Formales, Arequipa, Peru

${ }^{70}$ Institute of Nuclear Physics PAN, Krakow, Poland

${ }^{71}$ University of Łódź, Faculty of High-Energy Astrophysics,Lódź, Poland

${ }^{72}$ Laboratório de Instrumentação e Física Experimental de Partículas - LIP and Instituto Superior Técnico - IST, Universidade de Lisboa - UL, Lisboa, Portugal

73 "Horia Hulubei” National Institute for Physics and Nuclear Engineering, Bucharest-Magurele, Romania

${ }^{74}$ Institute of Space Science, Bucharest-Magurele, Romania

75 University Politehnica of Bucharest, Bucharest, Romania

${ }^{76}$ Center for Astrophysics and Cosmology (CAC), University of Nova Gorica, Nova Gorica, Slovenia

${ }^{77}$ Experimental Particle Physics Department, J. Stefan Institute, Ljubljana, Slovenia

${ }^{78}$ Universidad de Granada and C.A.F.P.E., Granada, Spain

${ }^{79}$ Instituto Galego de Física de Altas Enerxías (IGFAE), Universidade de Santiago de Compostela, Santiago de Com- 
postela, Spain

${ }^{80}$ IMAPP, Radboud University Nijmegen, Nijmegen, The Netherlands

${ }^{81}$ Nationaal Instituut voor Kernfysica en Hoge Energie Fysica (NIKHEF), Science Park, Amsterdam, The Netherlands

${ }^{82}$ Stichting Astronomisch Onderzoek in Nederland (ASTRON), Dwingeloo, The Netherlands

${ }^{83}$ Universiteit van Amsterdam, Faculty of Science, Amsterdam, The Netherlands

${ }^{84}$ University of Groningen, Kapteyn Astronomical Institute, Groningen, The Netherlands

${ }^{85}$ Case Western Reserve University, Cleveland, OH, USA

${ }^{86}$ Colorado School of Mines, Golden, CO, USA

${ }^{87}$ Department of Physics and Astronomy, Lehman College, City University of New York, Bronx, NY, USA

${ }^{88}$ Louisiana State University, Baton Rouge, LA, USA

${ }^{89}$ Michigan Technological University, Houghton, MI, USA

${ }^{90}$ New York University, New York, NY, USA

${ }^{91}$ Pennsylvania State University, University Park, PA, USA

92 University of Chicago, Enrico Fermi Institute, Chicago, IL, USA

93 University of Delaware, Department of Physics and Astronomy, Bartol Research Institute, Newark, DE, USA

94 University of Wisconsin-Madison, Department of Physics and WIPAC, Madison, WI, USA

${ }^{a}$ Fermi National Accelerator Laboratory, Fermilab, Batavia, IL, USA

${ }^{b}$ Max-Planck-Institut für Radioastronomie, Bonn, Germany

${ }^{c}$ School of Physics and Astronomy, University of Leeds, Leeds, United Kingdom

${ }^{d}$ Colorado State University, Fort Collins, CO, USA

$e$ now at Hakubi Center for Advanced Research and Graduate School of Science, Kyoto University, Kyoto, Japan

$f$ also at University of Bucharest, Physics Department, Bucharest, Romania 


\section{The Telescope Array Collaboration}

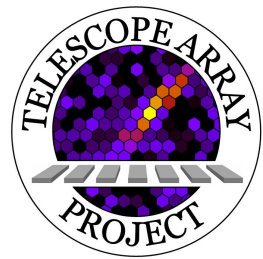

R.U. Abbasi ${ }^{1,2}$, T. Abu-Zayyad ${ }^{1,2}$, M. Allen ${ }^{2}$, Y. Arai $^{3}$, R. Arimura ${ }^{3}$, E. Barcikowski ${ }^{2}$, J.W. Belz ${ }^{2}$, D.R. Bergman ${ }^{2}$, S.A. Blake ${ }^{2}$, I. Buckland ${ }^{2}$, R. Cady ${ }^{2}$, B.G. Cheon ${ }^{4}$, J. Chiba ${ }^{5}$,

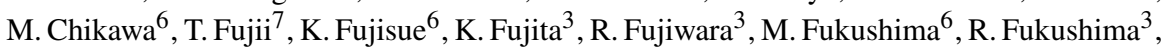
G. Furlich ${ }^{2}$, R. Gonzalez ${ }^{2}$, W. Hanlon ${ }^{2}$, M. Hayashi ${ }^{8}$, N. Hayashida ${ }^{9}$, K. Hibino ${ }^{9}$, R. Higuchi ${ }^{6}$,

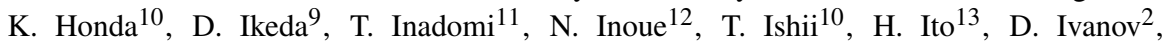
H. Iwakura ${ }^{11}$, A. Iwasaki ${ }^{3}$, H.M. Jeong ${ }^{14}$, S. Jeong ${ }^{14}$, C.C.H. Jui ${ }^{2}$, K. Kadota ${ }^{15}$, F. Kakimoto ${ }^{9}$, O. Kalashev ${ }^{16}$, K. Kasahara ${ }^{17}$, S. Kasami ${ }^{18}$, H. Kawai ${ }^{19}$, S. Kawakami ${ }^{3}$, S. Kawana ${ }^{12}$, K. Kawata ${ }^{6}$, I. Kharuk ${ }^{16}$, E. Kido ${ }^{13}$, H.B. Kim ${ }^{4}$, J.H. Kim ${ }^{2}$, J.H. Kim ${ }^{2}$, M.H. Kim ${ }^{14}$, S.W. Kim ${ }^{14}$, Y. Kimura ${ }^{3}$, S. Kishigami ${ }^{3}$, Y. Kubota ${ }^{11}$, S. Kurisu ${ }^{11}$, V. Kuzmin ${ }^{16}$, M. Kuznetsov ${ }^{16,20}$, Y.J. Kwon ${ }^{21}$, K.H. Lee ${ }^{14}$, B. Lubsandorzhiev ${ }^{16}$, J.P. Lundquist ${ }^{2,22}$, K. Machida ${ }^{10}$, H. Matsumiya ${ }^{3}$, T. Matsuyama ${ }^{3}$, J.N. Matthews ${ }^{2}$, R. Mayta ${ }^{3}$, M. Minamino ${ }^{3}$, K. Mukai ${ }^{10}$, I. Myers ${ }^{2}$, S. Nagataki ${ }^{13}$, K. Nakai ${ }^{3}$, R. Nakamura ${ }^{11}$, T. Nakamura ${ }^{23}$, T. Nakamura ${ }^{11}$, Y. Nakamura ${ }^{11}$, A. Nakazawa ${ }^{11}$, E. Nishio ${ }^{18}$,

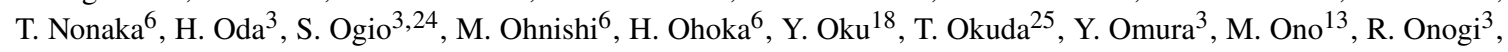
A. Oshima ${ }^{3}$, S. Ozawa ${ }^{26}$, I.H. Park ${ }^{14}$, M. Potts ${ }^{2}$, M.S. Pshirkov ${ }^{16,27}$, J. Remington ${ }^{2}$, D.C. Rodriguez ${ }^{2}$, G.I. Rubtsov ${ }^{16}$, D. Ryu ${ }^{28}$, H. Sagawa ${ }^{6}$, R. Sahara ${ }^{3}$, Y. Saito ${ }^{11}$, N. Sakaki ${ }^{6}$, T. Sako ${ }^{6}$, N. Sakurai ${ }^{3}$, K. Sano ${ }^{11}$, K. Sato ${ }^{3}$, T. Seki ${ }^{11}$, K. Sekino ${ }^{6}$, P.D. Shah ${ }^{2}$, Y. Shibasaki ${ }^{11}$, F. Shibata ${ }^{10}$, N. Shibata ${ }^{18}$, T. Shibata ${ }^{6}$, H. Shimodaira ${ }^{6}$, B.K. Shin ${ }^{28}$, H.S. Shin ${ }^{6}$, D. Shinto ${ }^{18}$, J.D. Smith ${ }^{2}$, P. Sokolsky ${ }^{2}$, N. Sone ${ }^{11}$, B.T. Stokes ${ }^{2}$, T.A. Stroman ${ }^{2}$, Y. Takagi ${ }^{3}$, Y. Takahashi ${ }^{3}$,

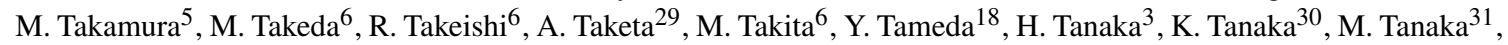
Y. Tanoue ${ }^{3}$, S.B. Thomas ${ }^{2}$, G.B. Thomson ${ }^{2}$, P. Tinyakov ${ }^{16,20}$, I. Tkachev ${ }^{16}$, H. Tokuno ${ }^{32}$, T. Tomida ${ }^{11}$, S. Troitsky ${ }^{16}$,

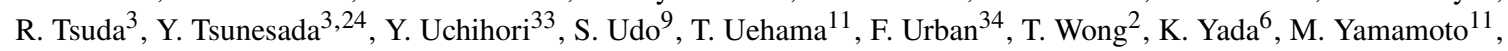
K. Yamazaki ${ }^{9}$, J. Yang ${ }^{35}$, K. Yashiro ${ }^{5}$, F. Yoshida ${ }^{18}$, Y. Yoshioka ${ }^{11}$, Y. Zhezher ${ }^{6,16}$, and Z. Zundel ${ }^{2}$

${ }^{1}$ Department of Physics, Loyola University Chicago, Chicago, Illinois, USA

${ }^{2}$ High Energy Astrophysics Institute and Department of Physics and Astronomy, University of Utah, Salt Lake City, Utah, USA

${ }^{3}$ Graduate School of Science, Osaka City University, Osaka, Osaka, Japan

${ }^{4}$ Department of Physics and The Research Institute of Natural Science, Hanyang University, Seongdong-gu, Seoul, Korea

${ }^{5}$ Department of Physics, Tokyo University of Science, Noda, Chiba, Japan

${ }^{6}$ Institute for Cosmic Ray Research, University of Tokyo, Kashiwa, Chiba, Japan

7 The Hakubi Center for Advanced Research and Graduate School of Science, Kyoto University, KitashirakawaOiwakecho, Sakyo-ku, Kyoto, Japan

${ }^{8}$ Information Engineering Graduate School of Science and Technology, Shinshu University, Nagano, Nagano, Japan

${ }^{9}$ Faculty of Engineering, Kanagawa University, Yokohama, Kanagawa, Japan

${ }^{10}$ Interdisciplinary Graduate School of Medicine and Engineering, University of Yamanashi, Kofu, Yamanashi, Japan

${ }^{11}$ Academic Assembly School of Science and Technology Institute of Engineering, Shinshu University, Nagano, Nagano, Japan

12 The Graduate School of Science and Engineering, Saitama University, Saitama, Saitama, Japan

${ }^{13}$ Astrophysical Big Bang Laboratory, RIKEN, Wako, Saitama, Japan

${ }^{14}$ Department of Physics, SungKyunKwan University, Jang-an-gu, Suwon, Korea

15 Department of Physics, Tokyo City University, Setagaya-ku, Tokyo, Japan

${ }^{16}$ Institute for Nuclear Research of the Russian Academy of Sciences, Moscow, Russia

${ }^{17}$ Faculty of Systems Engineering and Science, Shibaura Institute of Technology, Minato-ku, Tokyo, Japan

18 Department of Engineering Science, Faculty of Engineering, Osaka Electro-Communication University, Neyagawashi, Osaka, Japan

${ }^{19}$ Department of Physics, Chiba University, Chiba, Chiba, Japan

${ }^{20}$ Service de Physique Théorique, Université Libre de Bruxelles, Brussels, Belgium

${ }^{21}$ Department of Physics, Yonsei University, Seodaemun-gu, Seoul, Korea

${ }^{22}$ Center for Astrophysics and Cosmology, University of Nova Gorica, Nova Gorica, Slovenia 
${ }^{23}$ Faculty of Science, Kochi University, Kochi, Kochi, Japan

${ }^{24}$ Nambu Yoichiro Institute of Theoretical and Experimental Physics, Osaka City University, Osaka, Osaka, Japan

${ }^{25}$ Department of Physical Sciences, Ritsumeikan University, Kusatsu, Shiga, Japan

${ }^{26}$ Quantum ICT Advanced Development Center, National Institute for Information and Communications Technology, Koganei, Tokyo, Japan

27 Sternberg Astronomical Institute, Moscow M.V. Lomonosov State University, Moscow, Russia

${ }^{28}$ Department of Physics, School of Natural Sciences, Ulsan National Institute of Science and Technology, UNIST-gil, Ulsan, Korea

${ }^{29}$ Earthquake Research Institute, University of Tokyo, Bunkyo-ku, Tokyo, Japan

${ }^{30}$ Graduate School of Information Sciences, Hiroshima City University, Hiroshima, Hiroshima, Japan

${ }^{31}$ Institute of Particle and Nuclear Studies, KEK, Tsukuba, Ibaraki, Japan

32 Graduate School of Science and Engineering, Tokyo Institute of Technology, Meguro, Tokyo, Japan

33 Department of Research Planning and Promotion, Quantum Medical Science Directorate, National Institutes for Quantum and Radiological Science and Technology, Chiba, Chiba, Japan

${ }^{34}$ CEICO, Institute of Physics, Czech Academy of Sciences, Prague, Czech Republic

${ }^{35}$ Department of Physics and Institute for the Early Universe, Ewha Womans University, Seodaaemun-gu, Seoul, Korea

\section{The Auger-TA Energy Spectrum Working Group}

Douglas R. Bergman, Olivier Deligny, Francesco Fenu, Toshihiro Fujii, Dmitri Ivanov, Isabelle Lhenry-Yvon, Ioana Mariş, Markus Roth, Francesco Salamida, Yoshiki Tsunesada, and Valerio Verzi 